\title{
ACTIVIDADES DE OCIO Y SU PRESENCIA EN LAS REDES SOCIALES EN JÓVENES POTENCIALMENTE VULNERABLES
}

\author{
LEISURE ACTIVITIES AND THEIR PRESENCE IN SOCIAL NETWORKS IN \\ POTENTIALLY VULNERABLE YOUTH
}

\section{ATIVIDADES DE LAZER E SUA PRESENÇA EM REDES SOCIAIS EM JOVENS POTENCIALMENTE VULNERÁVEIS}

\author{
M.a Ángeles VALDEMOROS SAN EMETERIO*, Rosa Ana ALONSO RUIZ* \\ \& Nuria CODINA MATA** \\ *Universidad de La Rioja, ${ }^{* *}$ Universitat de Barcelona
}

PALABRAS CLAVE: redes sociales

formación profesional básica jóvenes vulnerables ocio interactividad interacción social
Fecha de recepción del artículo: 22.VII.2017 Fecha de revisión del artículo: 14.1X.2017 Fecha de aceptación final: 20.XI.2017

\begin{tabular}{|c|c|}
\hline $\begin{array}{l}\text { PALABRAS CLAVE: } \\
\text { redes sociales } \\
\text { formación } \\
\text { profesional básica } \\
\text { jóvenes vulnerables } \\
\text { ocio } \\
\text { interactividad } \\
\text { interacción social }\end{array}$ & $\begin{array}{l}\text { RESUMEN: La atención a los colectivos en riesgo de exclusión social en referencia a la alfabeti- } \\
\text { zación digital ha de tener mayor protagonismo en la investigación educativa y social. El objetivo } \\
\text { del estudio es analizar la presencia y difusión en las redes sociales de las actividades de ocio } \\
\text { de un colectivo de jóvenes potencialmente vulnerable, relacionándolo con el sexo y las áreas } \\
\text { geográficas de residencia. La muestra ascendió a } 140 \text { estudiantes españoles de Formación Pro- } \\
\text { fesional Básica. Se aplicó un cuestionario estructurado en diferentes bloques temáticos, del } \\
\text { que se seleccionaron los } 3 \text { ítems que indagaban en el tema objeto de estudio. Se llevaron a } \\
\text { cabo análisis descriptivos e inferenciales. Los resultados manifiestan que un } 62.8 \% \text { difunden } \\
\text { sus actividades de ocio en las redes sociales, siendo las actividades físicas las experiencias más } \\
\text { compartidas por este colectivo. Los chicos publican en mayor medida actividades festivas. Son } \\
\text { los norteños y quienes residen en el centro de España los que más comunican en las redes so- } \\
\text { ciales las actividades físicas y los levantinos quienes más comparten las experiencias culturales. } \\
\text { Las conclusiones de este estudio han proporcionado claves que optimizan la educación del ocio } \\
\text { digital de los estudiantes de Formación Profesional Básica para un uso constructivo y crítico de } \\
\text { las redes sociales, lo que contribuirá a la alfabetización digital de este colectivo y, por ende, a } \\
\text { minimizar las posibilidades de pertenecer a escenarios de riesgo. }\end{array}$ \\
\hline
\end{tabular}

CONTACTAR CON LOS AUTORES: M.a Ángeles Valdemoros San Emeterio. Universidad de La Rioja. Departamento de Ciencias de la Educación. Área de Teoría e Historia de la Educación. maria-de-los-angeles.valdemoros@unirioja.es.

INSTITUCIONES: El texto que presentamos se vincula al proyecto de investigación «De los tiempos educativos a los tiempos sociales: la construcción cotidiana de la condición juvenil en una sociedad de redes. problemáticas específicas y alternativas pedagógico-sociales» (Proyecto Coordinado EDU 2012-39080- C07-00) y al subproyecto «De los tiempos educativos a los tiempos sociales: la cotidianidad familiar en la construcción del ocio físico-deportivo juvenil» (EDU 2012- 39080-C07- 05), cofinanciado en el marco del plan nacional I+D+i con cargo a una ayuda del Ministerio de Economía y Competitividad, y por el Fondo Europeo de Desarrollo Regional (FEDER, 2007-2013). También la investigación ha contado con la ayuda puente a proyectos de investigación de la universidad de La Rioja (Ref: APPI 16/09). 


\begin{tabular}{|c|c|}
\hline $\begin{array}{l}\text { KEY WORDS: } \\
\text { social networks } \\
\text { basic vocational } \\
\text { training } \\
\text { vulnerable youth } \\
\text { leisure } \\
\text { interactivity } \\
\text { social interaction }\end{array}$ & $\begin{array}{l}\text { ABSTRACT: Attention to groups at risk of social exclusion concerning digital literacy must } \\
\text { play a greater role in educational and social research. The objective of the study is to analyze } \\
\text { the presence and dissemination in the social networks of the leisure activities of a collective } \\
\text { of potentially vulnerable youth, relating it to sex and the geographic areas of residence. The } \\
\text { sample was made up of } 140 \text { Spanish students of Basic Vocational Training. A questionnaire } \\
\text { structured in different thematic blocks was applied, from which we selected } 3 \text { items that } \\
\text { explored the topic under study. Descriptive and inferential analyses were carried out. The re- } \\
\text { sults show that } 62.8 \% \text { disseminate their leisure activities on the social networks, with physical } \\
\text { activities being the experiences most frequently shared by this group. The boys publish more } \\
\text { festive activities. The northerners and the residents of the center of Spain communicate more } \\
\text { physical activities in social networks, and the easterners share more cultural experiences. } \\
\text { The findings of this study have provided keys to optimize the digital leisure education of stu- } \\
\text { dents of Basic Vocational Training in the critical and constructive use of social networks, which } \\
\text { would contribute to the digital literacy of this collective and, therefore, would help minimize } \\
\text { the likelihood of their belonging to risky scenarios. }\end{array}$ \\
\hline $\begin{array}{l}\text { PALAVRAS-CHAVE: } \\
\text { redes sociais } \\
\text { treinamento } \\
\text { vocacional básico } \\
\text { juventude vulnerável } \\
\text { lazer interatividade } \\
\text { interação social }\end{array}$ & $\begin{array}{l}\text { RESUMO: A atenção aos grupos em risco de exclusão social em relação à alfabetização digital } \\
\text { deve ter um papel maior na pesquisa educacional e social. O objetivo do estudo é analisar a } \\
\text { presença e difusão nas redes sociais das atividades de lazer de um grupo de jovens potencial- } \\
\text { mente vulneráveis, relacionando-o com o sexo e as áreas geográficas de residência. A amostra } \\
\text { totalizou } 140 \text { estudantes espanhóis de Formação Profissional Básica. Um questionário estru- } \\
\text { turado foi aplicado em diferentes blocos temáticos, dos quais foram selecionados os } 3 \text { itens } \\
\text { que investigaram o tópico em estudo. Foram realizadas análises descritivas e inferenciais. Os } \\
\text { resultados mostram que } 62,8 \% \text { divulgam suas atividades de lazer nas redes sociais, sendo as } \\
\text { atividades físicas as experiências mais compartilhadas por este grupo. Os rapazes publicam } \\
\text { atividades mais festivas. São os habitantes do norte e os que residem no centro da Espanha } \\
\text { que mais comunicam nas redes sociais as atividades físicas e Levantinos que compartilham as } \\
\text { experiências mais culturais. As conclusões deste estudo forneceram chaves que otimizam a } \\
\text { educação digital de lazer dos alunos da Formação Profissional Básica para um uso construtivo } \\
\text { e crítico das redes sociais, o que contribuirá para a alfabetização digital deste grupo e, por- } \\
\text { tanto, para minimizar as possibilidades de pertencer a cenários de risco. }\end{array}$ \\
\hline
\end{tabular}

\section{Introducción}

El tándem conceptual ciencia/conciencia adquiere protagonismo en la sociedad del siglo XXI (Fernández-Buey, 2005) y reclama la atención de los colectivos vulnerables en el marco de una sociedad digital (Fuente-Cobo, 2017), lo que implica que constructos como el de exclusión social han de tener mayor presencia en las agendas de política social nacionales e internacionales (García-Blanco, 2016).

La alfabetización digital, desde un enfoque positivo y resiliente, y como apoyo a políticas de prevención, viabiliza oportunidades para abandonar ambientes de riesgo (Haenens, Vandoninck \& Donoso, 2013), por lo que se hace necesario utilizar su alto potencial como mecanismo de empoderamiento para el colectivo más vulnerable (Vaquero, 2013).

Estudios previos han puesto en evidencia el acceso, el impacto social y las bondades del uso de internet (Cloquell, 2015; Ochaita, Espinosa \& Gutiérrez, 2011); los jóvenes son el principal colectivo que crea y edita sus propios espacios virtuales, estableciéndose en verdaderos consumidores de comunicación digital y de manejo de las redes sociales virtuales (Colás, González \& De Pablos, 2013; Espinar \& González, 2009; Llamas \&
Pagador, 2010; Subrahmanyam, Greenfield \& Michikyan, 2015).

Recientemente, Valdemoros, Sanz y Ponce de León (2017) constatan que las redes sociales son la experiencia de ocio digital primordial de los jóvenes de educación secundaria postobligatoria. En esta línea, Almansa, Fonseca y Castillo (2013) refieren cómo los jóvenes conforman sus tipologías mediante las redes sociales, y Muros, Aragón y Bustos (2013) verifican que chatear, compartir fotos y jugar son los usos principales que los jóvenes hacen de las mismas.

Se confirma que lo que más impulsa a los jóvenes al uso de las redes sociales es el reconocimiento social (Almansa et al., 2013; Bernal \& Ángulo, 2013; Colás et al., 2013) y que las características de las amistades que los jóvenes establecen a través de las redes sociales se relacionan con sus comportamientos de ocio (Marks, de la Haye, Barnett \& Allender, 2015).

También es una evidencia que la exclusión se acrecienta en la Sociedad de la Información (Tezanos, 2001) porque se vincula a carencias tanto educativas como relacionales y de oportunidad, estando estrechamente relacionada con el concepto de "brecha digital", es decir, con las posibilidades de acceso a dispositivos tecnológicos y redes de comunicación, así como a su correcto uso, 
dado que es una de las condiciones capitales para la plena participación social en nuestra Sociedad Red (Fuente-Cobo, 2017).

Una de las bondades de la alfabetización digital es que permite a los colectivos vulnerables diseñar estrategias de e-inclusión y e-facilitación con el fin de recobrar sus habilidades para la interacción social y la participación cívica (Abad, 2014; Cáceres, Brändle \& Ruiz, 2015); en este sentido, puede hablarse de estrategias de empoderamiento en el colectivo de jóvenes que les capacitan para el uso de tecnologías de producción que les posibilitan visibilizar iniciativas, reivindicaciones y causas que de otra manera no tendrían oportunidad de hacerse patentes (Aguaded \& Sánchez, 2013; Saorín y Gómez, 2014), lo que viabiliza el ciberactivismo y la cibersolidaridad mediante las tecnologías sociales.

Es escasa la literatura científica que examina el uso que los jóvenes vulnerables realizan de las redes sociales; algunos autores (Melendro, García \& Goig, 2016) certifican que es similar al del resto de la población juvenil, si bien enfatizan como prioritario atender a ciertos elementos preocupantes que deberán considerarse en la acción social y educativa con este colectivo; en primer lugar, y en relación con el sexo, la evidencia de que las jóvenes vulnerables hacen un uso menor del ocio digital social si se compara con las chicas que pertenecen a colectivos no vulnerables; también, que la frecuencia e intensidad en su uso es mayor en los jóvenes vulnerables, así como su uso en soledad; y, finalmente, la menor capacidad de este colectivo para transformar la información recibida por este medio en auténtico capital cultural.

\section{Justificación y objetivos}

La Ley Orgánica para la Mejora de la Calidad de la Educación (LOMCE, 2013) justifica la introducción de la Formación Profesional Básica en la necesidad de reducir las tasas de abandono escolar temprano y luchar contra la exclusión social propiciada por las altas tasas del mismo. También el Real Decreto 127/2014 precisa que surgen los ciclos de Formación Profesional Básica como medida para facilitar la permanencia del alumnado en el sistema educativo y ofrecerles mayores posibilidades para su desarrollo personal y profesional.

La investigación que nos ocupa se focaliza en estudiantes españoles de Formación Profesional Básica (FPB) por considerar que estos ciclos son cursados por un colectivo de jóvenes potencialmente vulnerable, ya que en su mayoría son chicos y chicas que nutren las cifras del fracaso escolar y que por la vía educativa ordinaria no completarían una titulación profesional reconocida, lo que les clasificaría, a priori, como grupo más vulnerable en los ámbitos personal, social y laboral (García, 2014; Sanjuán-Roca \& Méndez-Lois, 2009).

El presente artículo tiene como objetivo analizar la presencia y difusión en las redes sociales de las actividades de ocio de un colectivo de jóvenes potencialmente vulnerable, relacionándolo con el sexo y las áreas geográficas de residencia. Esto permitirá conocer mejor los modos de interacción social de los estudiantes de FPB y ayudará a la tipificación de las actividades de ocio que más valoran, lo que facilitará claves que optimicen la educación de su ocio digital para un uso constructivo y crítico de las redes sociales, lo que exige de formación para gestionar gran cantidad de información compartida en entornos virtuales (García Valcárcel \& Tejedor, 2010).

\section{Metodología}

\subsection{Población y muestra}

La población se configura por 73.523 alumnos que cursan Formación Profesional Básica del estado español inscritos en el curso 2013-2014, siendo la muestra de 140 participantes, con un error muestral de $\pm 2,3$ y un nivel de confianza del $95 \%$.

En su mayoría (65\%) tienen edades comprendidas entre los 17 y 18 años, siendo el $23,6 \%$ mujeres $(n=33)$ y el $76,4 \%$ hombres $(n=107)$.

Se efectuó un muestreo aleatorio simple, intentando conservar una afijación proporcional en cada una de las 6 zonas en las que se fraccionó el territorio español, a lo que se añade Ceuta y Melilla (Tabla 1). 


\begin{tabular}{|l|l|}
\hline \multicolumn{2}{|c|}{ Tabla 1. Agrupación territorial para la muestra } \\
\hline Denominación/ Localización & \multicolumn{1}{c|}{ Comunidades y/o provincias } \\
\hline A1-Noreste & Cataluña, Aragón (excepto Teruel) y Baleares \\
\hline A2-Levante & Comunidad Valenciana, Murcia y Albacete \\
\hline A3-Sur & Andalucía, Islas Canarias, Ceuta y Melilla \\
\hline A4-Centro & $\begin{array}{l}\text { Madrid, Castilla-La Mancha (excepto Albacete), Castilla-León (excepto León, Palencia y } \\
\text { Burgos), Cáceres y Teruel }\end{array}$ \\
\hline A5-Noroeste & Galicia, Asturias y León \\
\hline A6-Norte & Cantabria, País Vasco, La Rioja, Navarra, Burgos y Palencia \\
\hline Fuente: elaboración propia &
\end{tabular}

\subsection{Variables}

Son 2 las variables que explican las características sociodemográficas de los estudiantes de FPB:

- Sexo del estudiante: variable dicotómica con dos categorías, hombre o mujer.

- Áreas territoriales: se fragmenta la nación, más Ceuta y Melilla, en 6 zonas, lo que facilita la zona geográfica de residencia, proporcionando 6 categorías: A1-Noreste; A2-Levante; A3-Sur; A4-Centro; A5-Noroeste; A6-Norte.

Y 3 las variables que precisan el ocio que este colectivo difunde en las redes sociales:

- Actividades de ocio más importantes: 8 categorías para identificar las actividades de ocio preferentes en los estudiantes de Formación Profesional Básica:

- Actividades de televisión o radio: ver la TV en general; ver en la TV programas de entretenimiento, programas deportivos, programas culturales o escuchar la radio.

- Actividades digitales: buscar información concreta en Internet; navegar por Internet sin un objetivo concreto; escribir mi propio blog o página web; compartir información; participar en chats, foros de discusión o comunidades virtuales; redes sociales; jugar a videojuegos; apuestas y juegos de azar online.

- Actividad física: actividades físicas artísticas y expresivas; actividades físicas en el medio natural; actividades físicas de cooperación y oposición; actividades de oposición uno contra uno; y actividades individuales.

- Turismo o excursionismo: viajar, hacer turismo, ir de campamentos, hacer excursiones al campo, a la montaña o a la playa, cazar o pescar.
- Actividades culturales: escuchar música, ver películas, leer libros que me gustan, ir al teatro, ir a museos, galerías o exposiciones, ir a conciertos o festivales, asistir a espectáculos deportivos, hacer alguna actividad artística, musical o literaria, etc.

- Fiestas o celebraciones: ir de copas, de "marcha" o a discotecas; salir a tomar algo con amigos; ir a las fiestas tradicionales de mi barrio o de mi ciudad; ayudar en la organización y desarrollo de las fiestas de mi barrio o fiestas tradicionales.

- Juegos (que no sean en el ordenador): juegos de mesa; billar, futbolín u otros juegos de salón; juegos de rol; otros juegos.

- Otras: cuidar animales, ir de compras, cuidar plantas, hacer manualidades, coleccionismo, asociacionismo o voluntariado.

- Número de actividades de ocio más importantes difundidas en las redes sociales: precisa cuántas de las tres actividades de ocio preferentes para el estudiante de FPB se difunden en las redes sociales.

- Tipo de actividades de ocio más importantes publicadas en las redes sociales.

\subsection{Instrumentos}

Se utilizó un cuestionario que se estructuraba en diversas unidades temáticas, por lo que acopiaba una información mucho más extensa de la que refleja este artículo. Fue validado mediante un juicio de expertos con 14 investigadores pertenecientes a 7 universidades españolas y una prueba piloto realizada en 8 comunidades autónomas.

Se emplean, para el presente estudio, los ítems 1 (sexo), 5 (situación familiar) y 28 (actividades que tienen presencia en las redes sociales). 


\subsection{Procedimiento}

Se aplicó el cuestionario aleatoriamente entre el alumnado perteneciente a algún curso de Formación Profesional Básica de los centros educativos de cada una de las 6 zonas geográficas establecidas, para lo que previamente se solicitó permiso tanto al Director General de Educación de cada Comunidad Autónoma como a los directores de los centros educativos, informándoles de los pormenores del estudio. Fueron dos investigadores convenientemente formados los responsables de pasar los cuestionarios, informando directamente a los estudiantes del modo correcto de responder al cuestionario, con el fin de reducir la mortalidad experimental.

\subsection{Análisis de datos}

Primero, se efectuó un análisis descriptivo que cuantificó las actividades de ocio que se difunden en las redes sociales y se registró las que tienen más presencia en las mismas. A continuación, se empleó la $t$ de Student para muestras independientes, con el fin de establecer la relación entre las características sociodemográficas de este colectivo y el número de actividades prioritarias de ocio que divulgan en las redes sociales. Finalmente, se calculó la relación entre las características sociodemográficas y el tipo de actividades principales de ocio que más comunican los estudiantes de Formación Profesional Básica, a través del análisis de tablas de contingencia, mediante los coeficientes Phi y $\vee$ de Cramer, respetando en todo momento un nivel de significatividad de $p<0.05$.

\section{Resultados}

Dos de cada tres estudiantes de FPB difunde en la red al menos una de sus tres actividades de ocio preferentes; el $42.1 \%$ comparte una de ellas, el $10.7 \%$ dos y el $10 \%$ tres.

Las actividades físicas se constituyen en la experiencia de ocio de los jóvenes españoles de Formación Profesional Básica más difundida en las redes sociales, pues un $20.7 \%$ afirma que comparte en la red estas prácticas, seguido de un $18.6 \%$ que declara publicar otras actividades, del $17.1 \%$ que afirma divulgar sus fiestas o celebraciones y del $14.3 \%$ de estudiantes de FPB que afirma difundir sus actividades culturales. Por contra, jugar a juegos de mesa o de salón (0.7\%), ver la televisión (3.6\%) y, finalmente, hacer turismo y compartir su ocio digital en Internet ( $4.3 \%$ cada uno) se establecen en las actividades menos anunciadas en la red por los estudiantes de Formación Profesional Básica (Figura 1).

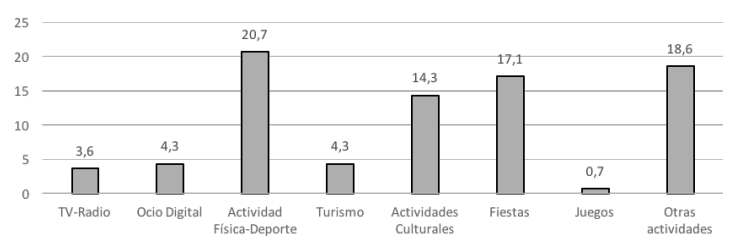

Figura 1. Frecuencia de estudiantes de FPB que publican cada tipo actividad de ocio principal en las redes sociales

Asimismo, se identifica que de los estudiantes de FPB que sitúan las actividades físicas en primer lugar de entre las tres preferentes, un $11.4 \%$ las publica en las redes sociales. Menor proporción se evidencia entre quienes posicionan las fiestas (7.9\%), otras actividades (6.4\%), las actividades culturales (5.7\%), ver la televisión (2.7\%), las actividades turísticas y digitales $(2.1 \%)$ y los juegos no digitales (0.7\%) como su primera experiencia de ocio preferente (Figura 2). En referencia a la difusión en redes sociales de la segunda actividad prioritaria vuelven a destacarse las actividades físicas (7.9\%).

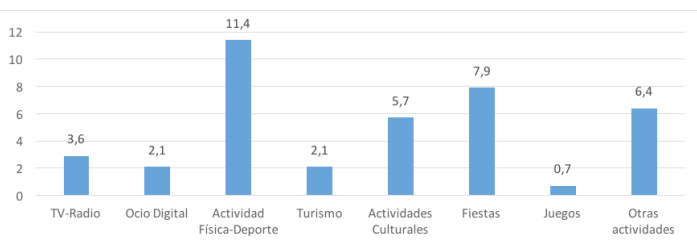

Figura 2. Frecuencia de estudiantes de FPB que publican su actividad principal de ocio en las redes sociales

No existen diferencias significativas en función del sexo a la hora de difundir y publicar actividades de ocio $\left(p=.001 ; \bar{x}_{m}=1.03 \pm 1.4748\right.$ vs $\bar{x}_{h}=0.91$ \pm 0.09346), ya que el análisis inferencial informa que la gran mayoría de actividades de ocio compartidas en las redes sociales son publicadas en la misma medida por chicas y chicos potencialmente vulnerabes, no mostrándose diferencias significativas en las referentes a la televisión ○ radio ( $p=.848 ; m=3 \%$ vs $h=3.7 \%$ ), las digitales $(p=.919 ; m=3 \%$ vs $h=4.7 \%)$, la actividad física $(p=.936$; $\mathrm{m}=21.2 \%$ vs $\mathrm{h}=20.6 \%$ ), las turísticas ( $p=.164 ; \mathrm{m}=0.0 \%$ vs $h=5.6 \%)$, las actividades culturales ( $p=.122$; $m=6.1 \%$ vs $h=16.8 \%$ ), los juegos no digitales ( $p=.577$; $\mathrm{m}=0.0 \%$ vs $\mathrm{h}=0.9 \%$ ), así como en la categoría de otras actividades $(p=.948 ; m=18.2 \%$ vs $h=18.7 \%)$. No sucede lo mismo con las actividades de fiesta ( $p=.005 ; m=33.3 \%$ vs $h=12.1 \%$ ), dado que se establecen en experiencias significativamente más difundidas por los chicos.

Tampoco se descubren diferencias significativas según la zona geográfica de residencia de estos jóvenes de Formación Profesional Básica, en 
cuanto a la propagación en las redes sociales de las actividades de televisión o radio, las de ocio digital, las turísticas, las fiestas y los juegos no digitales, ya que todas son igualmente publicadas en todas las áreas residenciales. Sin embargo, cuando se refieren a las actividades físicas ( $V$ de Cramer=.311; $p=.019)$, las actividades culturales ( $V$ de Cramer=.302; $p=.026$ ) y otras actividades ( $V$ de Cramer=.409; $p=.000$ ) sí se revelan diferencias significativas, débiles o moderadas, entre las distintas zonas.

Los jóvenes en riesgo de exclusión del norte son quienes más comunican en las redes sociales sus actividades físicas, seguidos por los de la zona centro. Los del noreste son los que menos informan de estas prácticas en la red, seguidos por los levantinos, los del noroeste y los del sur (Tabla 2).

Tabla 2. Tabla cruzada actividades físicas compartidas en las redes sociales por áreas territoriales

\begin{tabular}{|c|c|c|c|c|c|c|}
\hline $\begin{array}{l}\text { Actividades físicas y } \\
\text { deportivas compartidas } \\
\text { en las redes sociales }\end{array}$ & A1-Noreste & A2-Levante & A3-Sur & A4-Centro & A5-Noroeste & A6-Norte \\
\hline No Recuento & 4 & 22 & 22 & 35 & 16 & 12 \\
\hline \% dentro de áreas & $100 \%$ & $95.7 \%$ & $88 \%$ & $68.6 \%$ & $88.9 \%$ & $63.2 \%$ \\
\hline Sí Recuento & $\mathrm{O}$ & 1 & 3 & 16 & 2 & 7 \\
\hline$\%$ dentro de áreas & ০\% & $4.3 \%$ & $12 \%$ & $31.4 \%$ & $11.1 \%$ & $36.8 \%$ \\
\hline
\end{tabular}

El alumnado de Formación Profesional Básica levantino es quien más difunde digitalmente sus experiencias culturales, seguido por los del noroeste y los del nordeste, respectivamente. $Y$ los que residen en el centro del territorio español son los que menos comparten estas prácticas, seguidos por los que habitan en el norte y en el sur, respectivamente (Tabla 3).

Tabla 3. Tabla cruzada ocio cultural compartido en las redes sociales por áreas territoriales

\begin{tabular}{|l|r|r|r|r|r|r|}
\hline $\begin{array}{l}\text { Ocio cultural compartido en } \\
\text { las redes sociales }\end{array}$ & A1-Noreste & A2-Levante & A3-Sur & A4-Centro & A5-Noroeste & A6-Norte \\
\hline $\begin{array}{l}\text { No Recuento } \\
\% \text { dentro de áreas }\end{array}$ & 3 & 16 & 22 & 49 & 13 & 17 \\
\hline $\begin{array}{l}\text { Sí Recuento } \\
\% \text { dentro de áreas }\end{array}$ & $75 \%$ & $69.6 \%$ & $88 \%$ & $96.1 \%$ & $72.2 \%$ & $89.5 \%$ \\
\hline$\vee$ de Cramer=302; $p=026$ & $25 \%$ & $30.4 \%$ & 3 & $2 \%$ & 5 & 2 \\
\hline
\end{tabular}

Finalmente, se destaca que es el colectivo en riesgo de vulnerabilidad del norte quien más difunde otras actividades por las redes sociales, seguidos a una distancia importante por los sureños, los del centro y los del noroeste, siendo los del noreste quienes menos las comunican virtualmente (Tabla 4). 
Tabla 4. Tabla cruzada otras actividades compartido en las redes sociales por áreas territoriales

\begin{tabular}{|c|c|c|c|c|c|c|}
\hline $\begin{array}{l}\text { Otras actividades } \\
\text { compartidas en las redes } \\
\text { sociales }\end{array}$ & Al-Noreste & A2-Levante & A3-Sur & A4-Centro & A5-Noroeste & A6-Norte \\
\hline No Recuento & 4 & 21 & 21 & 44 & 16 & 8 \\
\hline \% dentro de áreas & $100 \%$ & $91.3 \%$ & $84 \%$ & $86.3 \%$ & $88.9 \%$ & $42.1 \%$ \\
\hline Sí Recuento & o & 2 & 4 & 7 & 2 & 11 \\
\hline \% dentro de áreas & ০\% & $8.7 \%$ & $16 \%$ & $13.7 \%$ & $11.1 \%$ & $57.9 \%$ \\
\hline
\end{tabular}

\section{Discusión y conclusiones}

Satisface constatar que los estudiantes de Formación Profesional Básica utilizan los medios digitales para publicar sus experiencias de ocio, mostrando que las redes sociales son una forma de comunicación y un nexo de unión importante para ellos (Almansa, Fonseca \& Castillo, 2013; Espinar \& González, 2009) lo que puede contribuir a su alfabetización digital y, en consecuencia, al logro de oportunidades para evadir contextos de riesgo (Haenens, Vandoninck \& Donoso, 2013).

El presente trabajo pone de manifiesto que las actividades físicas son las más difundidas en la red por los estudiantes de Formación Profesional Básica, seguidas de la participación en fiestas y celebraciones, en contraposición con las referidas a jugar a juegos de mesa o de salón, ver la televisión, hacer turismo y compartir ocio digital, que se establecen en el tipo de experiencias menos publicadas.

En este colectivo potencialmente vulnerable no se encuentran diferencias entre chicos y chicas a la hora de compartir la mayoría de las actividades de ocio en las redes sociales, salvo cuando se hace referencia a las de tipo festivo, en las que son los chicos quienes significativamente más las difunden; lo que podría situarse en sintonía con lo que Colás et al. (2013) constatan en alusión a que son los varones quienes más persiguen ser reconocidos por los demás mediante la divulgación de sus actividades en la red.

Nuestra investigación descubre que existen diferencias en el tipo de experiencias de ocio que el colectivo en riesgo de exclusión publica en la red, según su lugar de residencia, pues constata que son los estudiantes del norte y centro de España los que más informan de sus actividades físicas en las redes sociales. En cuanto a las actividades culturales difundidas en la red, son los levantinos quienes más las comparten, seguidos de los estudiantes domiciliados en las zonas noroeste y noreste. Sería de interés profundizar en futuros estudios en las posibles relaciones causales entre esta realidad y la oferta de ocio existente en cada área geográfica del estado español.

Durante la presente investigación se ha mantenido la prudencia de considerar a los estudiantes de Formación Profesional Básica como colectivo potencialmente vulnerable, por constatarse que son chicos y chicas con altos índices de fracaso escolar, que por la vía educativa ordinaria no completarían una titulación profesional reconocida, siendo susceptibles de quedar abocados a un mayor fracaso personal, social y laboral (García, 2014; Sanjuán-Roca \& Méndez-Lois, 2009). No obstante se considera que para dotar de mayor rigor científico al estudio que nos ocupa debería ampliarse la muestra a otros grupos en situación de riesgo de exclusión social no pertenecientes a este nivel educativo.

Debido a la escasez de investigación al respecto, sería de gran interés para la comunidad científica comprobar si la situación de los jóvenes potencialmente vulnerables es similar al del resto de la población juvenil, tal y como han indagado Melendro et al. (2016), quienes focalizan ciertos elementos preocupantes a considerar al comparar colectivos vulnerables y no vulnerables, en concreto en lo relacionado con un mayor uso en soledad de estos dispositivos en los chicos en riesgo de exclusión y su dificultad para transformar la información recibida por estos medios en verdadera riqueza cultural. De ahí que a los profesionales de la educación se nos encomiende la tarea de educar en el ocio digital para un uso responsable de las redes sociales (García Valcárcel \& Tejedor, 2010). 


\section{Referencias bibliográficas}

Abad, L. (2014). Diseño de programas de e-inclusión para alfabetización mediática de personas mayores. Comunicar, 42, 173-180. DOI: https://doi.org/10.3916/C42-2014-17

Aguaded, J.I., \& Sánchez, J. (2013). El empoderamiento digital de niños y jóvenes a través de la producción audiovisual. adComunica. Revista de estrategias, tendencias e innovación en comunicación, 5, 175-196. DOI: https://doi. org/10.6035/2174-0992.2013.5.11

Almansa, A., Fonseca, O., \& Castillo, A. (2013). Redes sociales y jóvenes. Uso de Facebook en la juventud colombiana y española. Comunicar, 4O(20), 127-135. DOI: http://dx.doi.org/10.3916/C40-2013-03-03

Bernal, C., \& Ángulo, F. (2013). Interacciones de los jóvenes andaluces en las redes sociales. Comunicar, 4O(20), 25-30. DOI: http://dx.doi.org/10.3916/C4O-2013-02-O2

Cáceres, M.D., Brändle, G., \& Ruiz, J.A. (2015). Hacia la construcción de una ciudadanía digital. Nuevos modelos de participación y empoderamiento a través de Internet. Prisma social, 15, 643-683. Recuperado de https://goo.gl/dw7Lml

Cloquell, A. (2015). Usos sociales de Internet entre los adolescentes españoles. Revista sobre la infancia y la adolescencia, 8, 1-14. DOI: doi.org/10.4995/reinad.2015.3649

Colás, P., González, T., \& de Pablos, J. (2013). Juventud y redes sociales: Motivaciones y usos preferentes. Comunicar 4O(20), 15-23. http://dx.doi.org/10.3916/C4O-2013-02-01

Espinar, E., \& González, M. J. (2009). Jóvenes en las redes sociales virtuales: un análisis exploratorio de las diferencias de género. Feminismo/s, 14, 87-105.

Fernández-Buey, F. (2005). Ciencia con conciencia. Quark, 36, 91-95. Recuperado de: http://quark.prbb.org/36/default. htm

Fuente-Cobo, C. (2017). Públicos vulnerables y empoderamiento digital: el reto de una sociedad e-inclusiva. El profesional de la información, 26(7), 5-12. DOI: https://doi.org/10.3145/epi.2017.ene.01

García, S. (2014). La investigación sobre el alumnado de los Programas de Cualificación Profesional Inicial ¿Punto de partida de la Formación Profesional Básica? Revista de Investigación en Educación, 12(2), 176-190.

García-Blanco, J.M. (2016). Los problemas teóricos y metodológicos del concepto de exclusión social. Una visión neofuncionalista. RIS. Revista internacional de sociología, 74 (2). DOI: https://doi.org/10.3989/ris.2016.74.2.029

García Valcarcel, A., \& Tejedor, F. J. (2010). Evaluación de procesos de innovación escolar basados en el uso de las TIC desarrollados en la Comunidad de Castilla y León. Revista de Educación, 352, 125-147.

Haenens, L., Vandoninck, S., \& Donoso, V. (2013). How to cope and build online resilience? EU Kids online. Recuperado de: http://eprints.lse.ac.uk/48115/

Ley Orgánica 8/2013, de 9 de diciembre, para la Mejora de la Calidad Educativa (LOMCE). (BOE, no 295, de 10 de diciembre).

Llamas, F., \& Pagador, I. (2014). Estudio sobre las redes sociales y su implicación en la adolescencia. Enseñanza \& Teaching: Revista interuniversitaria de didáctica, 32(7), 43-57. DOI: http://dx.doi.org/10.14201/et20143214357

Marks, J., de la Haye, K., Barnett, L.M., \& Allender, S. (2015). Friendship Network Characteristics Are Associated with Physical Activity and Sedentary Behavior in Early Adolescence. Plos One, 10(12), e0145344. DOI: 10.1371/journal. pone.0145344

Melendro, M., García, F.J., \& Goig, R. (2016). El uso de las TIC en el ocio y la formación de los jóvenes vulnerables. Revista española de pedagogía, 263, 71-89.

Muros, B., Aragón, Y., \& Bustos, A. (2013). La ocupación del tiempo libre de jóvenes en el uso de videojuegos y redes. Comunicar, 4O(20), 31-39. DOI: http://dx.doi.org/10.3916/C40-2013-02-03

Ochaita, E., Espinosa, M. A., \& Gutiérrez, H. (2011). Las necesidades adolescentes y las nuevas tecnologías de la información y la comunicación. Revista de Estudios de Juventud, 92, 183-192.

Real Decreto 127/2014, de 28 de febrero, por el que se Regulan aspectos específicos de la Formación Profesional Básica de las Enseñanzas de Formación Profesional del Sistema Educativo, se aprueban catorce títulos profesionales básicos, se fijan sus currículos básicos y se modifica el Real Decreto 1850/2009, de 4 de diciembre, sobre Expedición de Títulos Académicos y Profesionales correspondientes a las enseñanzas establecidas en la Ley Orgánica 2/2006, de 3 de mayo, de Educación (BOE, no 55, de 5 de marzo).

Sanjuan, M.M., \& Méndez-Lois, M. J. (2009). La innovación formativa a través de los Programas de Cualificación Profesional Inicial. Innovación Educativa, 19, 215-223.

Saorín, T., \& Gómez, J.A. (2014). Alfabetizar en tecnologías sociales para la vida diaria y el empoderamiento. Anuario ThinkEPI, 8, 342-348. Recuperado de https://goo.gl/pLVrVE

Subrahmanyam, K., Greenfield, P. M., \& Michikyan, M. (2015). Comunicación electrónica y relaciones adolescentes. Una actualización de las investigaciones existentes. Infoamérica: Iberoamerican Communication Review, 9, 115-130.

Tezanos, J.F. (2001). La sociedad dividida. Estructura de clases y desigualdades en las sociedades tecnológicas. Madrid: Biblioteca Nueva. 
Valdemoros, M.A., Sanz, E., \& Ponce de León, A. (2017). Ocio digital y ambiente familiar en estudiantes de Educación Postobligatoria. Comunicar, 5O(25), 99-108. DOI: http://dx.doi.org/10.3916/C50-2017-09

Vaquero, E. (2013). Estudio sobre la resiliencia y las competencias digitales de los jóvenes adolescentes en situación de riesgo de exclusión social. Universidad de Lleida [Tesis doctoral].

\section{CÓMO CITAR ESTE ARTÍCULO}

Valdemoros, M.A., Alonso, R.A., \& Codina, N. (2018). Actividades de ocio y su presencia en las redes sociales en jóvenes potencialmente vulnerables. Pedagogía Social. Revista Interuniversitaria, 31, 71-80. DOI: 10.7179/PSRI_2018.31.06

\section{DIRECCIÓN COMPLETA DE LOS AUTORES}

M.a Ángeles Valdemoros San Emeterio. Profesora Titular de Universidad. Universidad de La Rioja. Departamento de Ciencias de la Educación. Área de Teoría e Historia de la Educación. maria-de-los-angeles.valdemoros@unirioja.es

Rosa Ana Alonso Ruiz. Profesora contratada laboral. Universidad de La Rioja. Departamento de Ciencias de la Educación. Área de Didáctica y Organización Escolar. rosa-ana.alonso@unirioja.es

Nuria Codina Mata. Profesora Titular de Universidad. Universidad de Barcelona. Departamento de Psicología Social y Psicología Cuantitativa. Área de Psicología Social. ncodina@ub.edu

\section{PERFIL ACADÉMICO}

M.a Ángeles Valdemoros San Emeterio. Doctora en Ciencias de la Educación por la Universidad de La Rioja. Especialista universitaria en Educación para la Salud y Experta universitaria en Animación Sociocultural. Profesora Titular en la Facultad de Letras y de la Educación de la Universidad de La Rioja. Directora de la revista Contextos Educativos. Miembro del equipo de investigación "Actividad Física y Deporte en el espacio y tiempo de Ocio" [AFYDO]. Miembro de la Red de Equipos de Investigación en Estudios de Ocio, "Red OcioGune". Investigadora en temas de Educación, Ocio, Familia, Valores y Actividad Física, participa en investigaciones de I+D+l, autora de 7 libros, y más de 60 capítulos de libro y artículos científicos. ORCID: http://orcid. org/0000-0002-7389-4039

Rosa Ana Alonso Ruiz. Doctora en Bases Psicológicas y Actividad Físico-Deportiva. Acción y Desarrollo, con calificación Sobresaliente "cum laude". Profesora asociada del Departamento de Ciencias de la Educación en la Universidad de La Rioja desde 2010 hasta 2015. En la actualidad profesora contratada interina. Miembro del equipo de investigación "Actividad Física y Deporte en el espacio y tiempo de Ocio" [AFYDO]. Miembro de la Red de Equipos de Investigación en Estudios de Ocio, "Red OcioGune". Investigadora en temas de Educación, Ocio, Familia, Valores y Actividad Física, participa en investigaciones de I+D+l, autora de una veintena de capítulos de libro y artículos científicos. ORCID: http://orcid.org/0000-0003-3215-578X 
Nuria Codina Mata. Profesora del Departamento de Psicología Social y Psicología Cuantitativa. Facultad de Psicología. Universidad de Barcelona. Su investigación psicosocial sobre el ocio, el tiempo libre, el ser y la identidad se integran dentro del estudio de la gestión del tiempo, el bienestar personal y el desarrollo óptimo. Así, como fenómenos de gestión psicosocial del tiempo estudia: el tiempo y las actividades de ocio, las experiencias de ocio, el disfrute y la autonomía en la práctica de actividades estructuradas, la procrastinación y las orientaciones temporales. Estas investigaciones se desarrollan marcándose como objetivo: explicar, comprender y orientar la intervención psicosocial. Es autora de más de 100 publicaciones científicas, ha dirigido y participado en numerosas investigaciones y ha sido profesora visitante en diversas universidades de Europa, Norteamérica y Sudamérica. ORCID: http://orcid.org/o000-0003-0280-3651 